\title{
How to Test a Medical Technology for Space: Trauma Sonography in Microgravity ${ }^{1}$
}

\author{
Shannon Melton ${ }^{\dagger}$, George Beck ${ }^{\dagger}$, Douglas Hamilton ${ }^{\dagger}$, M.D., Ph.D., F.R.C.P.(C.), \\ Rosa Chun§, M.D., Ashot Sargsyan ${ }^{\dagger}$, M.D. and \\ Andrew W. Kirkpatrick ${ }^{*}$, M.D., F.R.C.S.(C.)
}

\begin{abstract}
Preventable trauma deaths in remote environments often result from inadequate diagnosis of thoracic and abdominal injuries. Full-time habitation of the International Space Station increases the risk of traumatic injury requiring intervention. This publication describes the evaluation of trauma sonography (TS) as a noninvasive, fast and effective space-based imaging tool for diagnosing intracavity hemorrhage or visceral leakage. The NASA Space Medicine Clinical Care Capability Development Project is using a four-phase approach: 1) identify terrestrial techniques for human diagnosis through literature or ground studies; 2) develop and test a model at 1-g if microgravity evaluations are required; 3) evaluate the model in microgravity (parabolic or shuttle flight); 4) implement the technology or technique for clinical use aboard the shuttle or space station. The Phase I literature review confirmed TS as the screening tool of choice for blunt trauma in most North American hospitals. In Phase II, an animal model was developed and tested for 1-g ground studies in which either fluid or air was injected into specific anatomical sites. Trained sonographers, using standard ultrasound techniques, successfully detected the fluid and air. The animal model was then prepared for the NASA KC-135 Microgravity Laboratory (Phase III). Injection and examination procedures were synchronized to the pull-in, 0-g and pull-out segments of the parabolic flight manoeuvres. Preliminary results indicate that trauma sonography is a clinically useful tool in microgravity. Phase IV efforts will address training, procedures, hardware, and data transfer requirements necessary to implement this technique for space.
\end{abstract}

\section{INTRODUCTION}

Throughout history, the activities associated with construction and exploration have been a cause of death and disability. The construction of the International Space Station (ISS) requires hundreds of hours of extra-vehicular activity (EVA), a particularly risky endeavour. The risks and severity of injury will be increased due to the number of person hours that

\footnotetext{
* To whom correspondence should be addressed: Assistant Director of Trauma Services, 3rd Floor, 855 W 10 Ave, Vancouver, British Columbia, Canada.

$\dagger$ Wyle Life Sciences, Houston, Texas, USA.

$\S$ Department of Anaesthesia, University of Toronto, Toronto, Canada.
}

will be spent in orbit and in particular EVA, the requirement for movement and construction of large masses, vehicle docking and refuelling, an advancing age of the astronaut population as highly specialised individuals are selected for specific missions, and an array of space induced physiological and pathological changes (1-4). Despite being weightless, objects retain their mass, and due to inertia can exert significant

$¥$ Trauma Services, Vancouver Hospital \& Health Sciences Centre, Vancouver, British Columbia, Canada.

1 The views expressed herein are those of the authors and do not reflect the official policies of the National Aeronautics and Space Administration, the Canadian Space Agency, nor other departments of the governments of Canada and the United States. 
forces if accelerated. Further, remarkably large mass objects can easily be accelerated in space. For example, the space arm, which effortlessly manipulates large structures in space, would buckle under these same loads on earth. Thus, objects, structures, or even individuals once accelerated in weightlessness could deliver crushing or lacerating blows $(5,6)$. This risk may well be accentuated by decreased perception of the risk of unintended acceleration, due to the lack of 'apparent' weight (6). Without weight as we know it on earth, humans are not as sensitive to inertial mass judgements, and consequently have shown decrements in proprioceptive abilities $(7,8)$.

NASA has published data which relate 'probable incidence versus impact on mission and health' for space missions and concluded that trauma is rated at the highest level (9). Penetrating injuries might occur during extravehicular activity, due to impacts from micro-meteorites; however a major penetration of a space suit is not likely to be a survivable occurrence (4). Conversely, blunt traumatic injuries may be more survivable and be amenable to diagnosis and treatment.

\section{TRAUMA CAPABILITIES REQUIREMENTS ABOARD THE ISS}

It is impossible to predict the exact scope of lifesaving surgical procedures that might be required on the ISS. In previous reviews of preventable deaths in isolated and rural treatment facilities, inadequate volume resuscitation, airway control, and diagnosis and interventions for intra-abdominal injuries, hemo/pneumothoraces, and treatable hemoperitoneum, were the main causes of preventable death (10-13). Given the extreme resource limitations on the ISS, it is logical to address those scenarios most likely to result in successful treatment outcomes. Thus, airway control and thoracoabdominal injuries are a prime focus for Space Medicine research.

On earth, overtly symptomatic or clinically suspicious abdominal and thoracic trauma are treated as surgical emergencies until proven otherwise, mandating immediate stabilization and transport of the patient to a facility that is able to deliver the appropriate standard of medical care. A similar approach is planned for the ISS; however, unnecessary evacuations will cause significant mission impacts and an exorbitant expense. It was previously estimated that an unscheduled medical evacuation to earth would cost at least 250 million U.S. dollars $(14,15)$. These cost estimates are now $\$ 500$ million.

Airway control and thoraco-abdominal injuries have been identified as prime targets for focussing trauma care. The assessment of the airway remains clearly a clinical task without reliance on diagnostic imaging. However, the diagnosis of thoraco-abdominal trauma is quite different. Physical examination is notoriously inaccurate in detecting intra-peritoneal injuries, especially with coexistent head and spinal injuries $(16,17)$. Despite continual emphasis on obtaining a rapid clinical diagnosis of hemo/pneumothorax, many cases are not initially suspected and are only diagnosed on a chest X-ray. Thus, terrestrial clinicians normally rely heavily on imaging techniques to assist in diagnosing intra-thoracic and intra-peritoneal injury. Techniques for imaging the abdomen and thorax include conventional radiology (X-ray), sonography and computed tomography (CT). Invasive diagnostic procedures include peritoneal lavage (DPL) and laparoscopy. X-ray and CT will not be available aboard the ISS. Laparoscopy and DPL are invasive procedures that require an experienced operator, yet the crew medical officer (CMO) on ISS will probably not be a clinician $(18,19)$. There is no requirement to have a physician on board the ISS at present, or in the near future. A non-physician CMO receives at most 40-60 hours of training, which would clearly be inadequate to deal definitively with major traumatic injuries.

Trauma sonography (TS) is a noninvasive, fast, safe, effective, repeatable, and tele-transmittable imaging tool that can screen for the presence of intra-cavitary (peritoneal, pericardial, and pleural) hemorrhage or visceral leakage (20-27). Abdominal Trauma Sonography (TS) has replaced DPL as the screening test of choice in many North American trauma centers (28), and can provide a definitive evaluation of many trauma patients in the appropriate settings. Ultrasound is often used to detect fluid collections in the pleural cavities and pericardium. The accurate imaging of the pleural spaces for the presence of fluid has been demonstrated in trauma settings (27).

The diagnosis of pneumothoraces with sonography is unorthodox, as the most common cause of indeterminate abdominal sonography in one focussed assessment with sonography for trauma (FAST) study was sub-cutaneous emphysema associated with thoracic injuries (29). The detection of air by sonography also appears initially somewhat paradoxical as the sonographic evaluation of the lung is hampered by the high acoustic reflectance of air-containing structures (30). Nevertheless, investigations have suggested that thoracic injuries including pneumothorax and hemothorax can also be reliably excluded with expanded TS. Preliminary human trials have also shown that TS can be effective in diagnosing a traumatic pneumothorax (31-33). These techniques are important because there will be no imaging modalities (X-ray or 
CT scan) on board the ISS other than ultrasound. Original plans for the Space Station specified medical care similar to that provided by the emergency, operating, and critical care units of a terrestrial Level III hospital, including emergency radiography (34). Currently, this is not feasible given current financial, mass, volume, and power restraints.

A state of the art ultrasound machine is manifested for the ISS, and could potentially provide the capability to perform TS. The essence of trauma sonography is the FAST exam, which does not look for individual organ injuries, but focuses on the detection of intra-peritoneal fluid (35). TS relies on the demonstration of sonolucent areas (fluid stripes) in typical anatomic locations. The localization of these fluid stripes is gravitationally determined in the 1-g environment. However, the behavior of intra-cavitary fluid remains poorly studied in weightlessness. If fluid does not localize to the expected or predicted terrestrial anatomical sites under microgravity conditions, erroneous interpretations of trauma sonograms might render the study nondiagnostic or even potentially misleading. Likewise, relying on an unproven technique to give a false reassurance that a pneumothorax is not present in a dyspneic astronaut, could be disastrous. It is thus crucial to ask, and then answer the question - can trauma sonography work in space?

\section{THE CLINICAL CARE CAPABILITY DEVELOPMENT PROGRAM}

As part of the Space Medicine Program at the Johnson Space Center, the Clinical Care Capability Development Program (CCCDP) is an endeavor to develop guidelines and practices to provide medical care onboard space vehicles such as the ISS. Prior to accepting any technology or recommending any technique it must be analyzed from the Space Medicine perspective. Procedures or techniques must be reviewed to determine the specific limiting factors that may be encountered in the space environment. Volatile anesthetics or intravenous fluids could not be used in the standard fashion, which requires gravity. Given the prohibitive costs involved in testing any procedure in orbit, the $\mathrm{KC}-135$ aircraft becomes an essential microgravity test-bed. This aircraft constitutes the NASA Reduced Gravity Research Platform, and allows investigators to simulate the weightlessness of space while remaining only a few miles off the earth's surface.

\section{MICROGRAVITY AND MICROGRAVITY RESEARCH LABORATORIES}

One of the most popular images of space is that of astronauts floating effortlessly across a capsule defying gravity. It is only with exploration class missions though, outside of the earth's orbit into the solar system and beyond, that astronauts will truly be free of the reach of gravity. The majority of mankind's space experience has occurred in orbital flight about the earth. In this environment, gravity exists but is counter-balanced by the centrifugal forces of the orbiting vehicle (36). 'Weightlessness' of an object is the result of the absence of acceleration vectors on this object. This is referred to as microgravity, in that gravity exists, but objects are effectively weightless. For over 30 years, parabolic flight has been used to generate brief periods of microgravity. By flying a parabolic profile, the contents and passengers of an aircraft are subject to weightlessness as they are experiencing no accelerative force vectors relative to the aircraft (37). The Russians have carried out a wide range of investigations in parabolic flight, including the first surgical-type experiments in weightlessness by performing laparotomies on rabbits in 1967 (38).

NASA uses the KC-135, a modified Boeing 707 aircraft as its microgravity research platform. Aboard the $\mathrm{KC}-135$, microgravity can be maintained for intervals of up to 30 -seconds. There are distinct disadvantages though, including; short windows of 0$\mathrm{g}$ followed by windows of 1.8-g during "pull-up" maneuvers, and the requirement to perform any lengthy procedures in a piece-meal fashion.

\section{MEDICAL CARE EVALUATION IN MICRO- GRAVITY}

A large body of published work, and an even larger amount of unpublished work has evaluated medical care in parabolic flight. Simulations performed aboard the KC-135 have demonstrated the ability to perform endotracheal intubation, mechanical ventilation, cardiopulmonary resuscitation (CPR), intravenous access with infusion of fluids and medications, intravenous anaesthetic techniques, central arterial, central venous and intracerebral pressure monitoring, debriding, suturing, and cleansing of wounds, splinting and casting of limbs, and the insertion of urinary and nasogastric catheters, using either training mannequins or animal models (1,6,39-43). Open surgical procedures on anaesthetised animal models have included exploratory laparotomy, mesenteric vein ligation and repair, and incision and repair of renal artery, carotid artery, and aorta, as well as laparotomy incision closure (44). Endoscopic procedures have included, laparoscopy and laparoscopic surgery techniques (45), and thoracoscopy and thorascopic techniques (46). Thus there is a well-established precedent for examining medical technology using parabolic flight as an 
analogue environment for space. The ability to carry out these procedures has, in part, depended on careful attention to restraint of the patient, operators, surgical and anaesthetic equipment, through a number of ingenious systems $(6,44-46)$.

\section{SPECIFIC TRAUMA SONOGRAPHY REQUIRE- MENTS}

Given real clinical requirements, an evaluation of the capability of ultrasound to diagnose medical conditions in weightlessness was required. The Operational Ultrasound Project was developed under the auspices of the CCCDP. The diagnosis of trauma being the first medical condition addressed. The trauma study was conceptualized and organized into two main portions, which consisted of an abdominal arm evaluating the FAST, and a thoracic arm looking at the ability to diagnose pneumo- and hemothoraces. In order to reproduce the physiologic conditions of a human in weightlessness, a porcine (i.e. pig) model was chosen. This model has been the human analogue used in the previous microgravity investigations looking at surgical and critical care procedures in parabolic flight.

\section{THE CLINICAL CARE CAPABILITY DEVELOPMENT PROGRAM PHILOSOPHY}

The CCCDP uses a four-phase approach to evaluating a medical problem and a linked potential solution: Phase I - identification of an efficacious terrestrial technique used on humans or recognition of a potential technique, Phase II - validation of the technique in 1-g (clinical practice or animal model), Phase III - validation of technique on a model in the 0 -g environment (microgravity analogue environment), Phase IV - operational implementation of the technique for use on humans in $0-\mathrm{g}$.

\section{EVALUATION OF TRAUMA SONOGRAPHY AS AN EXAMPLE OF THE FOUR-PHASED CCCDP EVALUATIVE PHILOSOPHY}

The process is by nature hierarchical, wherein there will be no commitment to progress to the next level if the results of the previous level do not support the investment of resources. If documentation or evidence of efficacy already exists, it will not always be necessary or practical for the CCCDP program to conduct a phase II trial. There may well be several components to Stages II and III, if ethical considerations mandate the study of an animal model prior to human studies in either or both stages. For the investigation of trauma sonography all phases were deemed necessary.

The initial stage (Stage I) consists of recognizing that a specific clinical problem on earth is usually diagnosed, prevented, or treated with a specific test or treatment. This may not be as easy in practice as in theory. There are unfortunately too many examples of unsubstantiated treatments that are strongly advocated on the basis of opinion as being essential, that soon become obsolete or even recognized to be harmful with further investigation or critical analysis. The routine application of military anti-shock trousers after injury or the rigid adherence to the maintenance of normal blood gases in the adult respiratory distress syndrome are only a few examples. The terrestrial use of trauma sonography (TS) appears to be a beneficial diagnostic tool without side effects, but even it might be superceded in the future by other diagnostic modalities, such as portable CT scanning. Currently TS warrants further evaluation for space, as sonography remains the only potential diagnostic modality aboard the ISS.

The next level of examination (Phase II) depends on whether the technique has been proven clinically in the course of routine terrestrial medicine. If a technology is currently utilized in everyday practice having documented effectiveness and safety, then phase II specifically focuses on refining the technique for the next phases of the CCCDP evaluation. For the detection of abdominal fluid, ground studies were only required for the validation of the porcine model as a legitimate model on which to perform abdominal sonography. When possible, human subjects would be used but it is sometimes necessary to use mannequins or animals models to test invasive techniques or procedures.

If a technology is unproven, the evaluative course can be even more complicated. The potential benefits are wider in scope though, as a new medical approach generated by a Space Medicine requirement may find wider terrestrial medical application. An example of such an unproven technology on earth, that has tremendous potential for operational Space Medicine, is the sonographic diagnosis of pneumothoraces. The diagnosis of pneumothorax has a high priority in Space Medicine, given the seriousness of a missed pneumothorax, the likely difficulty a non-clinican would have in making a clinical diagnosis with a stethoscope in a noisy environment, and the danger in instituting invasive chest drainage by an inexperienced operator. Although initially appearing paradoxical as air has a such high acoustic reflectance and does not transmit sound waves, the sonographic absence of the normal pleural movements has been successfully used to infer the presence of a pneumothorax (47-50). To investigate this potential, both animal and human 


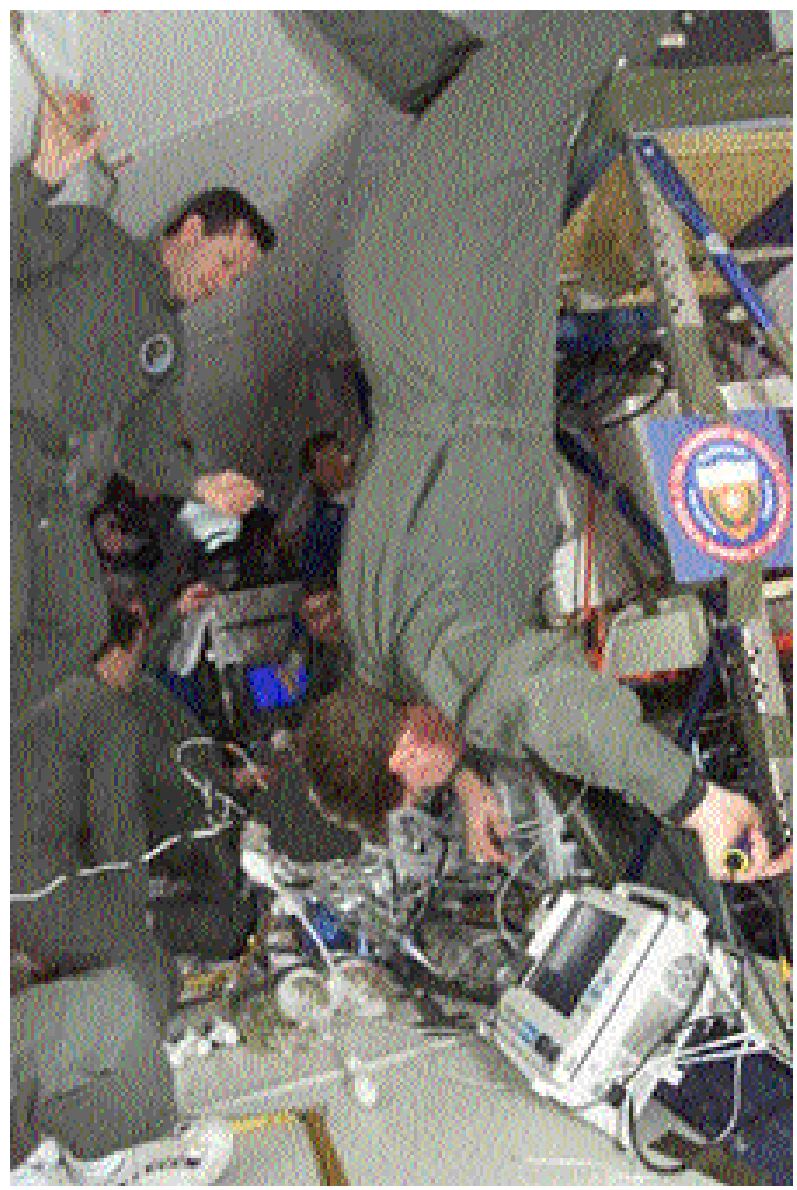

Figure 1.The microgravity research environment in parabolic flight aboard the KC-135 aircraft.

studies were undertaken in a normal gravity prior to any evaluation in weightlessness.

Human studies were being carried out evaluating the efficacy of sonography in detecting pneumothoraces in trauma victims at the Detroit Receiving Hospital in Detroit, Michigan. These results have recently been presented and show the reliable detection of pneumothoraces and $100 \%$ specificity in ruling out the presence of a pneumothorax (51). A similar study is underway with CT scan correlation at the Vancouver General Hospital. Preliminary results have corroborated the Detroit studies, and suggest that the sonographic evaluation of the chest may be more accurate than the radiographic examination of the supine chest in detecting pneumothoraces after trauma (S. Nicolaou personal communication). The promise of these terrestrial studies strengthened the need to critically evaluate the performance of thoracic sonography in weightlessness.

Simultaneously, animal investigations were carried out during the Ground Rehearsal phase of this project in Vancouver, British Columbia. These investigations validated the porcine model, showing that after a pneumothorax had been induced in an anesthetized animal, the sonographers could detect its presence. After treatment of the pneumothorax with a chest tube, the normal pleural motion (ruling out the presence of a pneumothorax) returned (32). This data justified the inclusion of an evaluation of the ability of sonography to detect pneumothoraces to be included as a separate arm of the trauma sonography study aboard the KC135 aircraft.

Given success in the phase II trials, the decision was made to proceed with an evaluation of trauma sonography in parabolic flight. Prior to the flight days aboard the $\mathrm{KC}-135$ aircraft, all procedures were first rehearsed in the animal laboratory at the Vancouver Hospital and Health Sciences Center. The animals were fully anesthetized and the ability to detect aliquots of fluid injected both centrally and into specific anatomic locations in the peritoneal cavity was verified and quantified. A laparoscopic examination was developed to place catheters at the specific anatomic locations that are examined in the traditional terrestrial FAST exam. These are Morrison's pouch, the splenorenal fossa, and the pouch of Douglas. For research purposes, catheters were also placed above the liver in the suprahepatic location, and another at the root of the small bowel mesentery. A major goal of this phase was to confirm the ability to perform successful sonography a short time after a pneumo-peritoneum had been created and then released. A time-sensitive protocol was also developed on the ground wherein the sequence of examination and injections of fluid and air were rehearsed in relation to the pull-in phase, assumed 25 second 0 -g phase, and in the pull-out phase to correspond to the anticipated flights. All data were video recorded with time synchronized voice recordings, thus chronicling the research activities.

A similar procedure was carried out for validation of both the hemothorax and the pneumothorax protocols. Baseline scans were recorded, followed by instrumentation of the pleural catheter, and then the injection of measured quantities of fluid. After the pneumothorax protocol had been examined, a similar evaluation of the feasibility of studying a hemothorax was carried out, facilitated by release of the intra-pleural air through a tube thoracostomy. Before each of these maneuvers, baseline scans were performed to document the state of the pleural space. These results confirmed the capabilities of trauma sonography to detect air and fluid in the pleural space (32). The ground lab data also 
generated a terrestrial control to compare the anticipated in-flight data.

\section{PHASE III, AN EVALUATION IN THE WEIGHTLESS CONDITIONS OF PARABOLIC FLIGHT}

Ultimately, four KC-135 microgravity flights were performed to determine the ability to diagnose abdominal and chest trauma in a porcine model using sonography equipment with both a high-definition broadband system (HDI-5000, ATL, USA) and a 2.4 kg. portable scanner (Sonosite-180, Sonosite Inc., USA). A flight-modified version of the same ATL ultrasound machine is manifested for the ISS. The protocols practiced and rehearsed in the ground lab were duplicated aboard the KC-135 (Figure 1). Prior to flight, catheters were either pre-positioned laparoscopically, or inserted blindly using a closed DPL technique in the abdomen to introduce air or fluid during KC-135 microgravity exposures. Fluid was injected during periods of microgravity to simulate the effects of traumatic injury in low earth orbit for the purpose of verification or modification of standard terrestrial sonographic diagnostic techniques. Laparoscopic and thoracoscopic visualization of these catheters and other surgical procedures were also performed. The ability to detect fluid and air in the thorax using sonography in microgravity was also investigated. A catheter was placed in the right thorax during a level flight period to introduce air and fluid during the following $0 \mathrm{~g}$ exposures. This simulated the effects of pneumothorax and hemothorax under microgravity conditions.

In addition to the validation of new microgravity sonographic imaging techniques, new critical care life support and monitoring equipment was evaluated. These technical evaluations examined a transport ventilator with an internal air compressor and blender, a multi-parameter critical care monitor, and an automatic external defibrillator. Air to ground telemetry was established for some instrumentation, (ultrasound, laparoscopic and physiologic data) and was monitored from the ground.

Formal and detailed review of the data is being carried out at present. All the videotapes of the sonographic findings have been analyzed into individual segments that correspond to either the hyper-gravity periods during the pull-in or pull-out maneuvers, or the actual period of weightlessness. These segments have been randomly rearranged and edited into a single videotape labeled only with numbers. Any reference to gravitational configuration was removed. A CD ROM describing the theory and rational behind the project and explaining a standardized scoring system was sent to noted experts in Trauma Sonography. In this way, both the investigators themselves and uninvolved experts were able to review the data in a blinded fashion. Ultimately a formal evaluation of these results will be submitted for peer reviewed publication.

\section{CONCLUSIONS}

The task at hand is the critical evaluation of the results from the blinded reviewers of both the abdomen and thoracic investigations. We subjectively believe that these techniques will be valuable tools in the limited Space Medicine armamentarium. Hopefully blinded impartial review will confirm this. A larger task will be that of phase 4 , the practical integration of this technology into the clinical pathways currently planned for Space Medicine contingencies. One of the major limiting factors will be the sophistication of the operators, who almost certainly will not be clinically current sonographers. To overcome this performance gap, interest is currently focused in evaluating automatic interpretation devices, and especially tele-medical and tele-sonographic capabilities and techniques. With continued expansion of mankind's exploration, and eventual habitation of Earth's orbit, the Lunar base and Mars, a dedicated and effective medical and surgical facility will be required. Planning and investigations should continue to address the needs for basic resuscitation and surgical intervention in low earth orbit, as a minimum requirement of care. Knowledge gained in these investigations will allow continued medical research and planning for future space exploration.

\section{REFERENCES}

1. Campbell MR, Billica RD. A review of microgravity surgical investigations. Aviation, Space and Environmental Medicine 1992; 63: 524-528.

2. Houtchens BA. Medical-Care systems for long duration space missions. Clinical Chemistry 1993; 39: 13-21.

3. Dons RF, Fohlmeister U. Combined injury syndrome in space-related radiation environments. Acta Space Research 1992; 12: 157-163.

4. Kirkpatrick AW, Campbell MR, Novinkov O, et al. Blunt trauma and operative care in microgravity. Journal of the American College of Surgeons 1997; 184: 441-453.

5. Houtchens B. System for the management of trauma and emergency surgery in Space. Final Report. Houston: NASA, Johnson Space Center; 1983. (NASA Grant NASW-3744).

6. McCuaig KE, Houtchens BA. Management of trauma and emergency surgery in space. Journal of Trauma 1992; 33: 610-625.

7. Ross H, Brodie E, BensonA. Mass discrimination during prolonged weightlessness. Science 1984; 225: 219-221. 
8. Ross H, Schwartz E, Emmerson P. The nature of sensimotor adaptation to altered g-levels: evidence from mass discrimination. Aviation, Space and Environmental Medicine 1987; 58: A148-A152.

9. Billica RD, Pool SL, Nicogossian AE. Crew Health Care Programs. In: Nicogossian AE, Huntoon CL, Pool SL, eds. Space Physiology and Medicine. 3rd ed. Philadelphia: Williams \& Wilkins; 1994: 402-423.

10. Veenema KR, Rodewald LE. Stabalization of rural multiple-trauma patients at level III emergency departments before transfer to a level I regional trauma center. Annals of Emergency Medicine 1995; 25: 175-181.

11. Certo TF, Rogers FB, Pilcher DB. Review of care of fatally injured patients in a rural state: 5 year follow-up. Journal of Trauma 1983; 23: 559-564.

12. Foley RW, Harris LS, Pilcher DB. Abdominal injuries in automobile accidents: review of care of fatally injured patients. Journal of Trauma 1977; 17: 611-615.

13. Houtchens BA. Major trauma in the rural mountain west. Annals of Emergency Medicine 1977; 6: 343-350.

14. Houtchens BA, Tonnesen AS. Minor surgery and anesthesia capabilities for space station health maintenance facility (HMF). Final report. NASA JSC Contract T-1419M. Houston, Texas, NASA Johnson Space Centre 1987.

15. Raymond CA. When medical help is really far away. JAMA 1988; 259: 2343-2344.

16. Wilson CB, Vidrine A, Rives JD. Unrecognized abdominal trauma in patients with head injuries. Annals of Surgery 1965; 161: 608-613.

17. Rodriguez A, Dupriest RW, Shatney CH. Recognition of intra-abdominal injury in blunt trauma victims. American Surgeon 1982; 48: 456-459.

18. Simmons SC. Telemedicine: a technology with space flight and terrestrial health care applications. 25th Internation Conference on Environmental Systems, San Diego, 1995.

19. Armstrong CW. Development of a telemedical workstation to support NASA. SAE tech paper series. 25th International Conference on Environmental Systems, San Diego, 1995.

20. Boulanger BR, McLellan BA, Brenneman FD, et al. Prospective evaluation of the superiority of a sonographybased algorithm in the assessment of blunt abdominal trauma. Journal of Trauma 1999; 47: 632-637.

21. Boulanger BR, McLellan BA, Brenneman FD, et al. Emergent abdominal sonography as a screening test in a new diagnostic algorithm for blunt trauma. Journal of Trauma 1996; 40: 1-7.

22. Rozycki GS, Ochsner MG, Feliciano DV, et al. Early detection of hemoperitoneum by ultrasound examination of the right upper quadrant: a multicenter study. Journal of Trauma 1998; 45: 878-883.

23. Wherrett LJ, Boulanger BR, McLellan BA, et al. Hypotension after blunt abdominal trauma: the role of emergent abdominal sonography in surgical triage. Journal of Trauma 1996; 41: 815-820.

24. Rozycki GS. Abdominal ultrasonography in trauma. Surgical Clinics of North America 1995; 75: 175-191.

25. Rozycki GS, Ballard RB, Feliciano DV, et al. Surgeonperformed ultrasound for the assessment of truncal injuries. Annals of Surgery 1998; 228: 557-567.

26. Rozycki GS, Ochsner MG, Jaffin JH, Champion HR. Prospective evaluation of surgeon's use of ultrasound in the evaluation of trauma patients. Journal of Trauma 1993; 34: 516-526.

27. Sisley A, Rozycki G, Ballard R, et al. Rapid detection of traumatic effusion using surgeon-performed ultrasound. Journal of Trauma 1998; 44: 291-297.

28. Boulanger BR, Kearney PA, Brenneman FD, et al. FAST utilization in 1999: result of a survey of North American trauma centers. American Surgeon. 2000; 66:1049-1055.

29. Boulanger BR, Brenneman FD, Kirkpatrick AW, et al. The indeterminate abdominal sonogram in multisystem blunt trauma. Journal of Trauma 1998; 45: 52-56.

30. Sistrom CL, Reiheld CT, Spencer BG, Wallace KK. Detection and estimation of the volume of pneumothorax using real-time sonography. AJR 1996; 166: 317-321.

31. Dulchavsky SA, Hamilton DR, Diebel LN, et al. Thoracic ultrasound diagnosis of pneumothorax. Journal of Trauma 1999; 47: 970-971.

32. Sargsyan AE, Hamilton D, Kirkpatrick AW, et al. Ultrasound evaluation of the magnitude of pneumothorax: a new concept. American Surgeon 2001; 67:232-236

33. Kirkpatrick AW, Ng A, Simons RK, Nicolaou. Sonographic diagnosis of a pneumothorax inapparent on plain chest radiography: confirmation by computed tomography. Journal of Trauma 2001; 50:75-752.

34. Space Station Projects Office. Requirements of an in-flight medical crew health care system (CHeCS) for Space Station. Houston: NASA Johnson Space Center; 1992. (Report No.: NASA JSC Document 31013 Rev D)

35. Scalea TM, Rodriguez A, Chiu WC, et al. Focused assessment with sonography for trauma (FAST): results from an international consensus conference. Journal of Trauma 1999; 46: 466-472.

36. Nicogossian AE, Robbins DE. Characteristics of the space environment. In: Nicogossian AE, Huntoon CL, Pool SL, eds. Space Physiology and Medicine. 3rd ed. Baltimore: Williams \& Wilkins, 1993: 50-62.

37. Greenisen MC, Edgerton VR. Human capabilities in the space environment. In: Nicogossian AE, Huntoon SL, Pool SL, eds. Space Physiology and Medicine. 3rd ed. Philadelphia: Lea \& Febiger, 1994: 50-62.

38. Yaroshenko GL, Terentyev BG, Mokrov MN. Characteristics of surgical intervention in conditions of weightlessness. Voyenno-Meditsinkiy Zhurnal 1967; 10: 69-70.

39. Markham SM, Rock JA. Microgravity testing a surgical isolation containment system for space station use. Aviatation, Space and Environmental Medicine 1991; 62: 691-693.

40. McCuaig K. Aseptic technique in microgravity. Surgical Gynecology and Obstetrics 1992; 175: 466-476.

41. McCuaig K. Surgical problems in space: an overview. Journal of Clinical Pharmacology 1994; 34: 513-517.

42. McCuaig K, Lloyd CW, Gosbee J, Synder WW. Simulation of blood flow in microgravity. American Journal of Surgery 1992; 164: 119-123.

43. Campbell MR, Billica RD, Johnston SL. Surgical bleeding in microgravity. Surgical Gynecology and Obstetrics 1993; 177: 121-125.

44. Campbell MR, Billica RD, Johnston SL. Animal surgery in microgravity. Aviation, Space and Environmental Medicine 1993; 64: 58-62.

45. Campbell MR, Billica RD, Jennings R, Johnston SL. Laparoscopic surgery in weightlessness. Surgical Endoscopy 1996; 10: 111-117.

46. Campbell MR, Kirkpatrick AW, Billica RD, et al. Endoscopic surgery in weightlessness. Surgical Endoscopy (in press).

47. Lichtenstein D, Meziere G, Biderman P, Gepner A. The comet-tail artifact: an ultrasound sign ruling out pneumothorax. Intensive Care Medicine 1999; 25: 383-388. 
48. Lichtenstein DA, Menu Y. A bedside ultrasound sign ruling out pneumothorax in the critically ill: lung sliding. Chest 1995; 108: 1345-1348.

49. Wernecke K, Galanski M, Peters PE, Hansen J. Pneumothorax: evaluation by ultrasound - preliminary results. Journal of Thoracic Imaging 1987; 2: 76-78.
50. Goodman TR, Traill ZC, Phillips AJ, et al. Ultrasound detection of pneumothorax. Clinical Radiology 1999; 54: 736739.

51. Dulchavsky SA, Schwarz KW, Hamilton DR, et al., Prospective evaluation of thoracic ultrasound in the detection of pneumothorax. J Trauma 2001; 50:201-205.

Shannon Melton has worked for Wyle Life Sciences in support of the manned space program since 1991 and is currently a systems engineer in the Advanced Projects Group of Medical Operations investigating and developing advanced clinical capabilities for space. George Beck is also at Wyle Laboratories as Advanced Projects Operational Medical Lead, responsible for managing the projects to augment the medical capability of the International Space Station. Douglas Hamilton, M.D., Ph.D., F.R.C.P.(C.) is a Flight Surgeon for Wyle Life Sciences at NASA Johnson Space Center supporting the Shuttle and Space Station Operations. He is also an electrical engineer and clinical consultant for the team developing the Shuttle-based telemedicine instrumentation system. Rosa Chun, M.D. is in her final year the Royal College of Physicians and Surgeons of Canada specialty training in Anesthesiology at the University of Toronto and is interested in measurement and outcome of clinical anesthesia. Ashot Sarsyan, M.D. is a Space Medicine physician at the Advanced Projects section of Wyle Life Sciences and has worked in the areas of clinical ultrasound and telemedicine. Andrew W. Kirkpatrick, M.D., F.R.C.S.(C.) is a general and trauma surgeon, multi-disciplinary intensivist, and Assistant Director of Trauma Services at the Vancouver General Hospital. He is also a trained military paratrooper, Flight Surgeon, and private multi-engine pilot, who consults to the National Aeronautics and Space Administration (NASA), Canadian Space Agency (CSA), and the Defence and Civil Institute of Environmental Medicine (DCIEM). 